\title{
REVIEW
}

\section{Plankton patchiness, turbulent transport and spatial spectra}

\author{
Peter J. S. Franks* \\ Scripps Institution of Oceanography, University of California, San Diego, La Jolla, California 92093-0218, USA
}

\begin{abstract}
Spatial variability of plankton is a well-known, though poorly characterized and understood, phenomenon. Several studies suggest that patches of plankton are essential to the growth and survival of some planktonic species; thus, any ability to predict the spatial patterns of plankton would significantly enhance our understanding of ocean ecosystems. Furthermore, models that accurately describe planktonic patchiness could permit a mechanistic understanding of the dynamics structuring the plankton. One such model is based on plankton being mixed in 3D isotropic turbulence, giving a $k^{-5 / 3}$ slope to the spatial spectrum of plankton variability. Many papers have been published that (often favorably) compare their data to this $k^{-5 / 3}$ model. Using a selection of papers published since 1993, I show, however, that the data in many of these studies have not been gathered on scales that allow comparison to the $\mathrm{k}^{-5 / 3}$ model. I then discuss whether planktonic data can even be used to test such a model: the model is continuum-based, while plankton are quanta at scales relevant to the turbulent scales. Still, many data sets resolving planktonic patchiness show a $k^{-5 / 3}$ slope of the spectrum, or at least show a biological spectrum with a different slope than the temperature, salinity, or velocity spectra. I discuss several aspects of biological data that might consistently bias the spectral slopes of planktonic spectra, and conclude that the spectral slope does not contain enough information to distinguish among alternate models of plankton patchiness. While this paper is not a critique of the $k^{-5 / 3}$ model or of the spectral method, it is a critique of the use of spectra of biological properties to test the $k^{-5 / 3}$ model. I hope that by pointing out some of the pitfalls of spectral analysis of biological data we might gain better insights into the physical-biological interactions creating microscale patchiness in the ocean.
\end{abstract}

KEY WORDS: Turbulence $\cdot$ Patchiness $\cdot$ Spectrum $\cdot$ Inertial subrange $\cdot$ Plankton $\cdot$ Variability Resale or republication not permitted without written consent of the publisher

\section{INTRODUCTION}

Ever since Ernst Haeckel put to rest Viktor Hensen's notion that plankton are uniformly distributed in the ocean, we have been searching for ways to measure, characterize, and understand the dynamics underlying plankton patchiness. Plankton patchiness has been shown to be a ubiquitous and important feature of oceanic ecosystems. Many organisms have been shown to exploit patches of food, and patch formation may be necessary for the sexual encounters among individuals of relatively rare species. If we can understand the physical and biological dynamics controlling plankton patchiness, we may gain some predictive ability concerning the scales, intensities, dura- tions, and ecological importance of this planktonic variability.

One powerful tool for quantifying the spatial scales and intensities of planktonic variability is Fourier or spectral analysis. In spectral analysis, a data series is decomposed into a series of sines and cosines with increasingly small wavelengths (high wave numbers) or short periods (high frequencies). The amplitude of the sine or cosine gives the intensity of variability at that scale. Commonly, the log of the spectral density, the variance or 'energy' given by the amplitude squared, of the property is plotted versus the log of the wavenumber or frequency. This is the spectrum.

In physical systems, the slope of the velocity spectrum is generally characteristic of the underlying 
dynamics. One particularly well-known example is the wavenumber spectrum of 3D turbulence. For a wavenumber $k$ (the wavenumber is $2 \pi /$ wavelength) the velocity spectrum has a shape given by $k^{-5 / 3}$, as discussed in more detail below. Models of plankton in physical flows have been developed that predict planktonic variability to have a spectrum with a slope of $k^{-\beta}$, where $\beta$ depends on the types of physical dynamics, and the relative strengths of physical and biological forces. In particular, one of the most wellknown models predicts that, in a turbulent flow, plankton should show a spectrum that has some portion that falls as $k^{-5 / 3}$. If we could gather appropriate data to test these models, we might gain an important element of understanding and predictive ability of planktonic patchiness in the ocean. The dynamics that determine the model spectral slope might reasonably be inferred to be controlling the spectral slope of the field data. If this is so, we now have a mechanistic understanding of the dynamics controlling planktonic variability.

A great deal of work has been undertaken in the last 3 decades formulating spectral models of plankton patchiness, and in particular gathering field data to test these models. As new instruments have been developed, new and better-resolved data sets have been generated. Comparison of these data to the spectral models has yielded interesting insights into the dynamics structuring the plankton. However, in the decades since the models were developed, the comparison of models and data has often been performed uncritically. In other words, do the data really represent a test of the model, and are the data appropriate to compare to the model? Below, I demonstrate that the data are often inappropriate for comparison to certain models, as they have been gathered on the wrong spatial scales. Furthermore, a comparison of spectral slopes of models and data does not represent a strong test of the model. There are a number of factors that can control the spectral slope of the data that cannot be accounted for in the model, and multiple combinations of dynamics can generate the same spectral slope. Here, I discuss several potential properties of data sets that are generally ignored in analyses of biological data, but which may significantly alter the spectral slope, giving spurious correlations with models.

I begin by describing the 3D model of the inertial subrange of turbulence, followed by a discussion of models of passive and reactive tracers in this flow. These models are the most commonly used for comparison to biological data, and it is worth understanding their genesis and limitations. I then analyze the data from 12 recently published papers to explore whether the data should be compared to 3D turbulence models, and whether the data test the models. Furthermore, it is useful to ask whether biological data can be used to test continuum models of planktonic distributions in isotropic turbulence. I then explore 2 alternate spectral models of plankton patchiness: (1) internal waves and (2) geostrophic turbulence. This is followed by an analysis of particular features of data sets that might give unrepresentative spectral slopes. It is my hope that by becoming more aware of the pitfalls of spectral analysis we might use it more critically and in conjunction with other analysis techniques to explore and understand plankton patchiness in the oceans.

\section{THE $\boldsymbol{k}^{-5 / 3}$ INERTIAL SUBRANGE MODEL}

In 1941, Kolmogorov put forward the hypothesis of an inertial subrange for 3D isotropic turbulence at high Reynolds numbers. He hypothesized that between the large scales at which turbulence is generated, and the smallest scales at which turbulence is dissipated, there should exist a range over which turbulent eddies lose their energy to successively smaller eddies, with little loss of energy to heat, until the smallest eddies are dissipated by viscosity. The only factors governing the velocities of the water motions at a given scale in this inertial subrange are the rate at which turbulent kinetic energy is put into the system (parameterized as the rate of turbulent kinetic energy being dissipated, $\varepsilon,\left[\mathrm{m}^{2} \mathrm{~s}^{-3}\right]$ ), and the length scale (given as the wavenumber, $k_{\text {, }}$ $\left.\left[\mathrm{m}^{-1}\right]\right)$. Based on this assumption, dimensional analysis leads to the energy spectrum given by:

$$
E(k)=\alpha \varepsilon^{2 / 3} k^{-5 / 3}
$$

where $\alpha$ is a scaling parameter. Thus, within the inertial subrange, the spectrum should fall off with increasing wavenumber with a slope of $k^{-5 / 3}$. A great deal of data from the atmosphere, ocean, and laboratory has confirmed Kolmogorov's hypothesis, and it is now considered a theory rather than a model.

The inertial subrange is constrained at the largest and smallest spatial scales. At the large scales, the turbulent eddies must work against stratification in causing mixing. Stratification is given by the buoyancy frequency, $N\left(\mathrm{~s}^{-1}\right)$ :

$$
N^{2}=-\frac{g}{\rho} \frac{\partial \rho}{\partial z}
$$

where $\rho$ is the density, $g$ the acceleration due to gravity, and $z$ the vertical coordinate. If the stratification is too strong (large $N$ ), the mixing cannot overcome it, and the turbulence will not be isotropic (e.g. Gargett et al. 1984). The largest turbulent eddies become flattened and anisotropic. The largest turbulent scales are usually parameterized by the Ozmidov scale, $L_{0}$, or the buoyancy wavenumber, $k_{\mathrm{b}}$ :

$$
L_{\mathrm{o}}=k_{\mathrm{b}}^{-1}=\sqrt{\varepsilon N^{-3}}
$$


In unusual situations such as deep convective mixing or exceptionally strong tidal mixing, $L_{0}$ can be as large as $100 \mathrm{~m}\left(\varepsilon \sim 10^{-5} \mathrm{~m}^{2} \mathrm{~s}^{-3}, N \sim 10^{-3} \mathrm{~s}^{-1}\right)$. In most of the ocean, most of the time, however, the largest turbulent scales are between about 10 and $100 \mathrm{~cm}\left(\varepsilon \sim 10^{-9}\right.$ to $10^{-8} \mathrm{~m}^{2} \mathrm{~s}^{-3}, N \sim 10^{-4}$ to $10^{-3} \mathrm{~s}^{-1}$ ). In wind-mixed layers, $\varepsilon$ tends to be between $10^{-8}$ and $10^{-6} \mathrm{~m}^{2} \mathrm{~s}^{-3}$ for winds of $<5$ to $15 \mathrm{~m} \mathrm{~s}^{-1}$, giving an $L_{\mathrm{o}}$ of 3 to $30 \mathrm{~m}$. Away from surface and bottom boundary layers, however, $L_{0}$ tends to be small due to weak mixing and relatively strong stratification.

The smallest turbulent scales are determined by the rate of turbulent kinetic energy input (again parameterized as $\varepsilon$ ), working against the loss of momentum through viscosity, $v\left(\mathrm{~m}^{2} \mathrm{~s}^{-1}\right)$. This is the Kolmogorov scale, $\eta$, and $k_{\mathrm{s}}$ is the Kolmogorov wavenumber:

$$
\eta=k_{\mathrm{s}}^{-1}=\left(v^{3} \varepsilon^{-1}\right)^{1 / 4}
$$

With a lot of turbulent kinetic energy, $\eta$ will be small for a given viscosity, generating energetic small-scale flows. If the viscosity is high, the smallest energetic scales will be larger. In most of the ocean, $\eta$ is about $1 \mathrm{~cm}$, though the energy-containing scales are somewhat larger (e.g. Lazier \& Mann 1989).

Thus, in 3D isotropic turbulence at high Reynolds number, an inertial subrange of turbulence should exist with a spectral slope of $k^{-5 / 3}$ of the energy spectrum between the buoyancy wavenumber, $k_{\mathrm{b}}$, at the largest scales, and the Kolmogorov wavenumber, $k_{\mathrm{s}}$ at the smallest scales. In most of the ocean, most of the time, the inertial subrange would be found between about 1 to $100 \mathrm{~cm}$, a 2 decade range. In exceptional circumstances, the inertial subrange may span up to 4 decades (0.01 to $100 \mathrm{~m}$ ). Gargett et al. (1984) suggested that the inertial subrange was found between about $10 k_{\mathrm{b}} \leq k \leq 0.1 k_{\mathrm{s}}$ in waters mixed by breaking nonlinear internal waves. In general, Gargett et al. (1984) found the strongest, most isotropic inertial subrange when $k_{\mathrm{s}} / k_{\mathrm{b}} \sim 1000$; that is, when turbulence was intense, creating a 3 decade range of spatial scales separating the buoyancy and Kolmogorov wavenumbers. With smaller ratios of $k_{\mathrm{s}} / k_{\mathrm{b}}$ (less energetic flows), the inertial subrange of velocity disappeared.

\section{$\boldsymbol{k}^{-5 / 3}$ passive tracer model}

Batchelor (1959) presented a theory describing the spectral shape given by variability of a passive tracer advected in a 3D isotropic turbulent flow. He assumed a tracer (such as temperature) with a source of variance at large scales and a dissipation of variance at small scales. The dissipation was analogous to the dissipation of turbulent kinetic energy, and is termed $\chi$ $\left(\mathrm{C}^{2} \mathrm{~s}^{-1}\right.$, where $\mathrm{C}$ is the unit of the tracer). Using the same dimensional arguments as Kolmogorov, Batchelor showed that there should be a region in the inertial subrange of turbulence in which the spectrum of temperature fluctuations is given by:

$$
E(k)=B \chi \varepsilon^{-1 / 3} k^{-5 / 3}
$$

where $B$ is a scaling parameter. Thus, the spectrum of temperature fluctuations in the inertial subrange was also predicted to have a $k^{-5 / 3}$ dependence on wavenumber. Depending on the ratio of the viscosity $v$ to the tracer diffusivity, $\gamma\left(\mathrm{m}^{2} \mathrm{~s}^{-1}\right)$, there may exist a region of $k^{-1}$ slope at the high-wavenumber (small-scale) end of the spectrum, the viscous-convective range. For our purposes, however, the main point is that the passive tracer should have a region of the variance spectrum showing a slope of $k^{-5 / 3}$ in a 3D isotropic turbulent flow, and this range should fall within the inertial subrange of turbulence. Gargett (1985) showed that temperature fluctuations in a measured, well-developed inertial subrange of turbulence $\left(k_{\mathrm{s}} / k_{\mathrm{b}} \sim 3000\right.$, strong mixing) did not follow a $k^{-5 / 3}$ slope, but rather showed a slope closer to $k^{-3 / 2}$. Curiously, temperature fluctuations in an anisotropic velocity field $\left(k_{\mathrm{s}} / k_{\mathrm{b}} \sim 100\right.$, weaker mixing) tended to have a more pronounced $k^{-5 / 3}$ slope. Gargett (1985) explained these features through intermittency of turbulence in the strong mixing case, and anisotropy (stratification effects causing horizontal stirring) of the water motions in the weaker mixing case.

\section{$k^{-5 / 3}$ plankton model}

In the early 1970s, a theory was developed predicting spectral slopes of plankton in turbulent flows (Denman \& Platt 1976, Denman et al. 1977). These early models were fashioned after models of Corrsin (1961), who studied chemical reactions in turbulent mixing chambers. Denman and his colleagues assumed a biological rate, $r\left(\mathrm{~s}^{-1}\right)$, along with the usual (but contestable) assumptions of a source of biological variance at large scales and dissipation of that variance at small scales at rate $\chi$. When combined with the other parameters of $k, \varepsilon$ and $v$, dimensional analysis led to the prediction of 3 regimes in the spectrum of planktonic variance. The low-wavenumber (large-scale) regime was dominated by biological growth, and the spectral slope was given by:

$$
E_{\mathrm{lw}}(k)=D \chi r^{-1} k^{-1}
$$

Here again, $D$ is a scaling parameter. In this largescale, low-wavenumber range, the spectrum is predicted to fall as $k^{-1}$.

The second regime was found in the inertial subrange of turbulence, where turbulent motions dominated the spectrum: 


$$
E_{\text {is }}(k)=B \chi \varepsilon^{-1 / 3} k^{-5 / 3}
$$

Here, like the passive tracer discussed by Batchelor (1959), the plankton variability should show a $k^{-5 / 3}$ dependence on wavenumber.

The third regime was found at the smallest scales (high wavenumbers), where a viscous-convective subrange might exist:

$$
E_{\mathrm{hw}}(k)=C \chi(v / \varepsilon)^{1 / 2} k^{-1}
$$

where $C$ is a scaling parameter. The spectral slopes of the 3 predicted regimes were thus $k^{-1}$ for the lowwavenumber, biologically dominated regime, $k^{-5 / 3}$ for the inertial subrange, and $k^{-1}$ in the viscous-convective regime at high wavenumbers.

Powell \& Okubo (1994) built on the earlier work of Denman by including plankton-plankton interactions (e.g. grazing) in their spectral model of plankton patchiness. They showed that in 3D turbulence, their model reproduced those of Denman if the plankton had no interactions with each other. In particular, the spectrum had a region of $k^{-5 / 3}$ slope in the inertial subrange of turbulence. However, if planktonic interactions

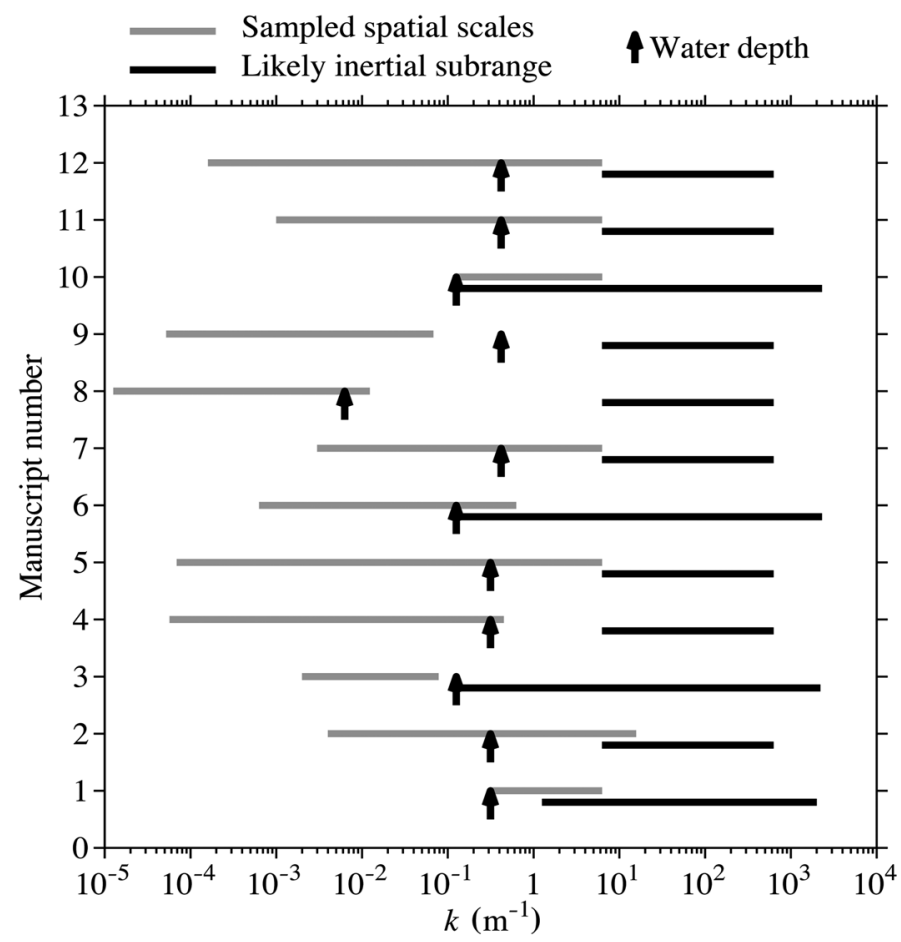

Fig. 1. Summary of data from 12 manuscripts published since 1993, indicating the range of spatial scales sampled (thick gray lines), the likely spatial range of the inertial subrange of 3D isotropic turbulence (thick black lines), and the water depth (arrows). In most cases there is little overlap between the scales sampled and the scales of the inertial subrange. For data sampled in time, I have used the authors' conversions to space were included, the spectral slope could be almost anything, including having discontinuities in the turbulent inertial subrange. This may have been the first suggestion that the spectral slope of plankton distributions did not have enough information to accurately diagnose the underlying dynamics.

\section{TESTS OF THE $\boldsymbol{k}^{-5 / 3}$ INERTIAL SUBRANGE PLANKTON MODEL}

Since Denman \& Platt's (1976) prediction of a $k^{-5 / 3}$ inertial subrange in the spectrum of plankton embedded in a 3D turbulent field, many studies have been published (and continue to be published) comparing their data to the $k^{-5 / 3}$ spectral model. It is important to ask, however, whether these data constitute a strong test of the $k^{-5 / 3}$ model, or, indeed, whether the data should be compared to the $k^{-5 / 3}$ model at all.

To make the point that the issues that I discuss below are current, I chose to analyze 12 papers published since 1993 in which a time-series or a spatial series of plankton distributions were compared to the $k^{-5 / 3}$ model (Tsuda et al. 1993, Mountain \& Taylor 1996, Seuront et al. 1996a,b, 1999, 2002, Wiebe et al. 1996, Seuront \& Lagadeuc 1997, 2001, Lovejoy et al. 2001a,b, Pershing et al. 2001). In some cases, the comparison of the data to the $k^{-5 / 3}$ model is quite ancillary to the main point of the paper, and any criticisms implied by the following analyses do not detract from the central points of their work. The data to be explored have been gathered in the open ocean and in coastal regions, with most of the data from coastal regions. Several of the papers discuss data gathered on Georges Bank, an area that is particularly turbulent due to tidal mixing (e.g. Yoshida \& Oakey 1996).

The papers to be analyzed include data collected by remote sensing, vertical profiling, horizontal transects, anchor stations, and sampling while drifting. The data include samples of dissolved nutrients, chlorophyll a fluorescence, and zooplankton. Zooplankton data were obtained using an optical particle counter or acoustic backscatter. For data collected as a time series, I have used the authors' own conversions to space (frequency to wavenumber).

To compare the planktonic data to the $k^{-5 / 3}$ model, the data must resolve the appropriate spatial scales, the inertial subrange. This inertial subrange lies between the Ozmidov scale, $L_{0}$, at the large end, and the Kolmogorov scale, $\eta$, at the small end. Except in particularly intense turbulence, the inertial subrange usually lies between scales of about 1 and $100 \mathrm{~cm}$. In Fig. 1, I have plotted the range of wavenumbers/spatial scales resolved by the various sampling programs, as well as an estimated range for the inertial subrange. To accu- 
rately estimate the inertial subrange would require knowledge of $\varepsilon, N$ and $v$, the dissipation rate of turbulent kinetic energy, the buoyancy frequency (the vertical density gradient), and the viscosity, respectively. Unfortunately, these values are not included in most of the papers. Given this lack of information, I estimated the inertial subrange to be 1 to $100 \mathrm{~cm}$ in most cases, and 0.3 to $5000 \mathrm{~cm}$ in the well-mixed waters over Georges Bank. In these tidally mixed waters, the turbulence mixes the water column from top to bottom, giving a maximal turbulent eddy size the same size as the water depth (about $50 \mathrm{~m}$ ) (but see Pershing et al. 2001 for an alternate explanation of the vertical circulation). This estimation of the location of the inertial subrange may be generous, based on the estimates of Gargett et al. (1984) who suggested that the inertial subrange would be found between about $10 k_{\mathrm{b}} \leq k \leq 0.1 k_{\mathrm{s}}$ (i.e. a much smaller spatial range than $k_{\mathrm{b}}$ to $k_{\mathrm{s}}$ ), though this estimate is for stratification-limited turbulence and may not be valid for turbulence influenced by the surface or bottom of the ocean.

It is quite apparent from Fig. 1 that, in most cases, the inertial subrange lies well outside the range of sampled spatial scales, or only overlaps slightly with the data. It is only in cases of extreme vertical mixing (e.g. Georges Bank) that the sampled scales overlap with the spatial scales of the turbulent inertial subrange by more than a decade. Interestingly, in these cases, the spectral slope of the fluorescence was statistically indistinguishable from $k^{-5 / 3}$ (e.g. Mountain \& Taylor 1996).

It is possible that my estimates of the range of the inertial subrange were too conservative. Perhaps the turbulent eddies were much larger than I allowed. The most unconservative estimate of the size of the largest eddies that could still be 3-dimensionally isotropic (statistically identical in every direction) is the depth of the water column; no isotropic eddy can be larger than the water column depth. Plotted in Fig. 1 is the water column depth given in each of the published studies. In several cases, the sampling is too coarse to resolve even eddies that would mix the water from top to bottom; in most cases, the sampling would resolve only about 1 decade of the largest possible inertial subrange. With these sampling constraints, there is little possibility of resolving the inertial subrange of turbulence. If the inertial subrange is not resolved by the sampling, it is inappropriate to compare the data to the $k^{-5 / 3}$ model.

\section{Dimensionality}

The Kolmogorov model of the inertial subrange is inherently 3D; isotropic turbulence implies that the structures (velocity, temperature, passive tracers) are statistically identical in every direction. However, we

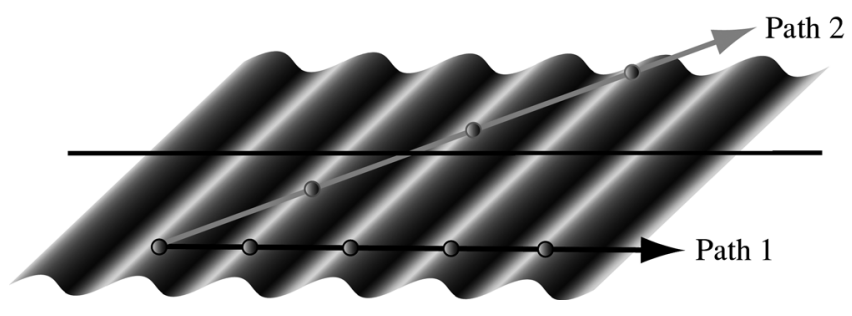

Fig. 2. 1D sampling through a 2D wave field; sampling along Path 1 (perpendicular to the crests) gives the correct wavenumber (the wavelength is indicated by the distance between the dots on the path). Sampling along Path 2 (at an angle to the crests) gives a lower wavenumber (dots are further apart), but the same amplitude as Path 1 . In the spectrum, the wave energy for Path 2 will have been aliased into a lower wavenumber (longer wavelength) than Path 1, which has the correct wavenumber

typically sample only along 1 dimension, or at the most 2 dimensions (as in remote sensing). Is it reasonable to compare data gathered in 1 dimension to a 3D model?

The problem with sampling a 3D field with a 1D sampling device is that the energy (variance, spectral density) of high-wavenumber features can be aliased into lower wavenumbers. A 2D analog of this problem can be illustrated with 1D sampling of a wave field (Fig. 2). Samples taken along a transect perpendicular to the crests of the waves will record an accurate wavelength and amplitude (energy, variance, or spectral density) for the waves. A transect taken at an angle to the crests will always record a longer wavelength (lower wavenumber), but the same amplitude. In the resulting spectrum, this energy will appear at a lower wavenumber than it should.

Hinze (1959) presented a transformation for unaliasing the energy from a $1 \mathrm{D}$ spectrum to allow comparison to a 3D spectral model. For a given 1D wavenumber spectrum $E_{1 \mathrm{D}}(k)$, the $3 \mathrm{D}$ spectrum $E_{3 \mathrm{D}}(k)$ can be obtained by:

$$
E_{3 \mathrm{D}}(k)=-k \frac{\partial}{\partial k} E_{1 \mathrm{D}}(k)
$$

Thus, multiplying the slope of the 1D spectrum by the wavenumber gives the 3D spectrum. For a 1D spectrum that is proportional to $k^{-n}$, the 3D spectrum is:

$$
E_{3 \mathrm{D}}(k)=-k \frac{\partial}{\partial k} k^{-n}=n k^{-n}
$$

giving a 3D spectral slope that is the same as the 1D spectrum. Thus, a spectrum that slopes as $k^{-5 / 3}$ in 1D should have the same slope in 3D. However, this only works if the 1D spectrum has no curvature in log-log space; that is, there is only $1 n$ ( $n$ is any number) and it is constant for the entire range of wavenumbers. As an example of what can happen when the 1D spectrum has curvature, I performed this transformation on the 


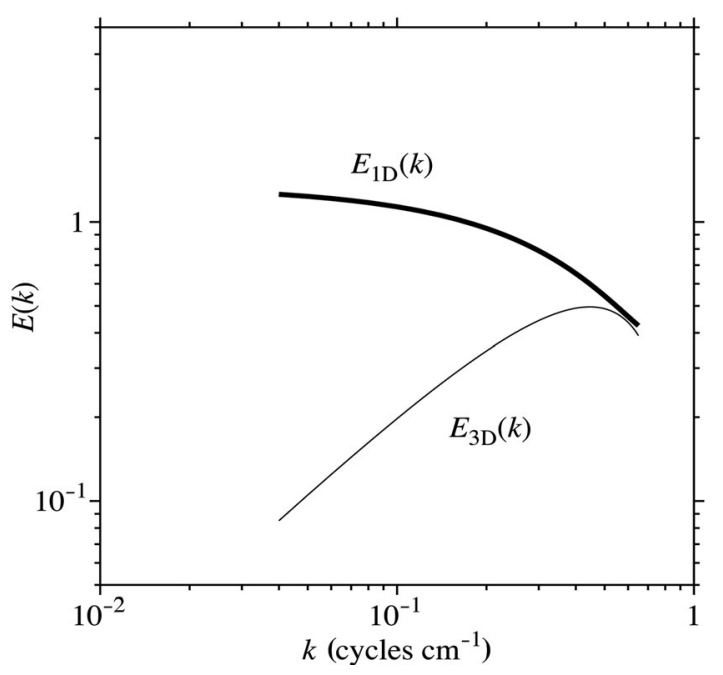

Fig. 3. An example of a conversion from a 1D spectrum $\left(E_{1 \mathrm{D}}(k)\right.$, thick line) to a 3D spectrum $\left(E_{3 \mathrm{D}}(k)\right.$, thin line) using Eq. (9). This conversion removes the low-wavenumber energy aliased by 1D sampling of high-wavenumber variability in an isotropic 3D field. In this case, the 1D spectrum monotonically decreased with wavenumber, while the 3D spectrum increased over most of its wavenumber range. Data from Franks \& Jaffe (2001)

spatial spectrum of fluorescence given in Franks \& Jaffe (2001, their Fig. 11) (Fig. 3). Even though the 1D spectrum monotonically decreases with increasing wavenumber (though with no suggestion of a $k^{-5 / 3}$ sloping region), the transformation to 3 dimensions gives a spectrum that increases over much of the wavenumber range. The transformation has removed spectral energy from the low wavenumbers and transferred it to higher wavenumbers, completely changing how we might interpret the spectral slopes. Even in isotropic turbulence, this transformation will tend to reduce the range of wavenumbers over which we find an inertial subrange by removing spectral energy from the low-wavenumber end of the spectrum (e.g. Gargett et al. 1984).

\section{Can the inertial subrange model be tested with biological data?}

The analyses presented above suggest that few data sets exist that can actually test the $k^{-5 / 3}$ model of plankton patchiness in 3D isotropic turbulence, since most data sets do not resolve the appropriate (small) spatial scales. However, an interesting problem arises when the small scales are resolved by appropriate instruments; plankton occur as quanta. Plankton are not a continuum as the $k^{-5 / 3}$ model requires. Plankters are individuals, and when they are viewed on scales around the high-wavenumber end of the inertial subrange, they appear as quanta rather than as a continuum.

To illustrate this problem, I use an image of phytoplankton fluorescence gathered with an imaging fluorometer, similar to the OSST (Franks \& Jaffe 2001). The fluorescence of phytoplankton was stimulated by a thin $(6.5 \mathrm{~mm})$ sheet of green laser light, and the fluorescence recorded by a sensitive CCD camera with a bandpass filter centered at $685 \mathrm{~nm}$. The camera and laser were mounted on a free-falling vehicle, and sequences of images acquired $80 \mathrm{~cm}$ below the vehicle as it sank at about $10 \mathrm{~cm} \mathrm{~s}^{-1}, 10 \mathrm{~km}$ off the coast of San

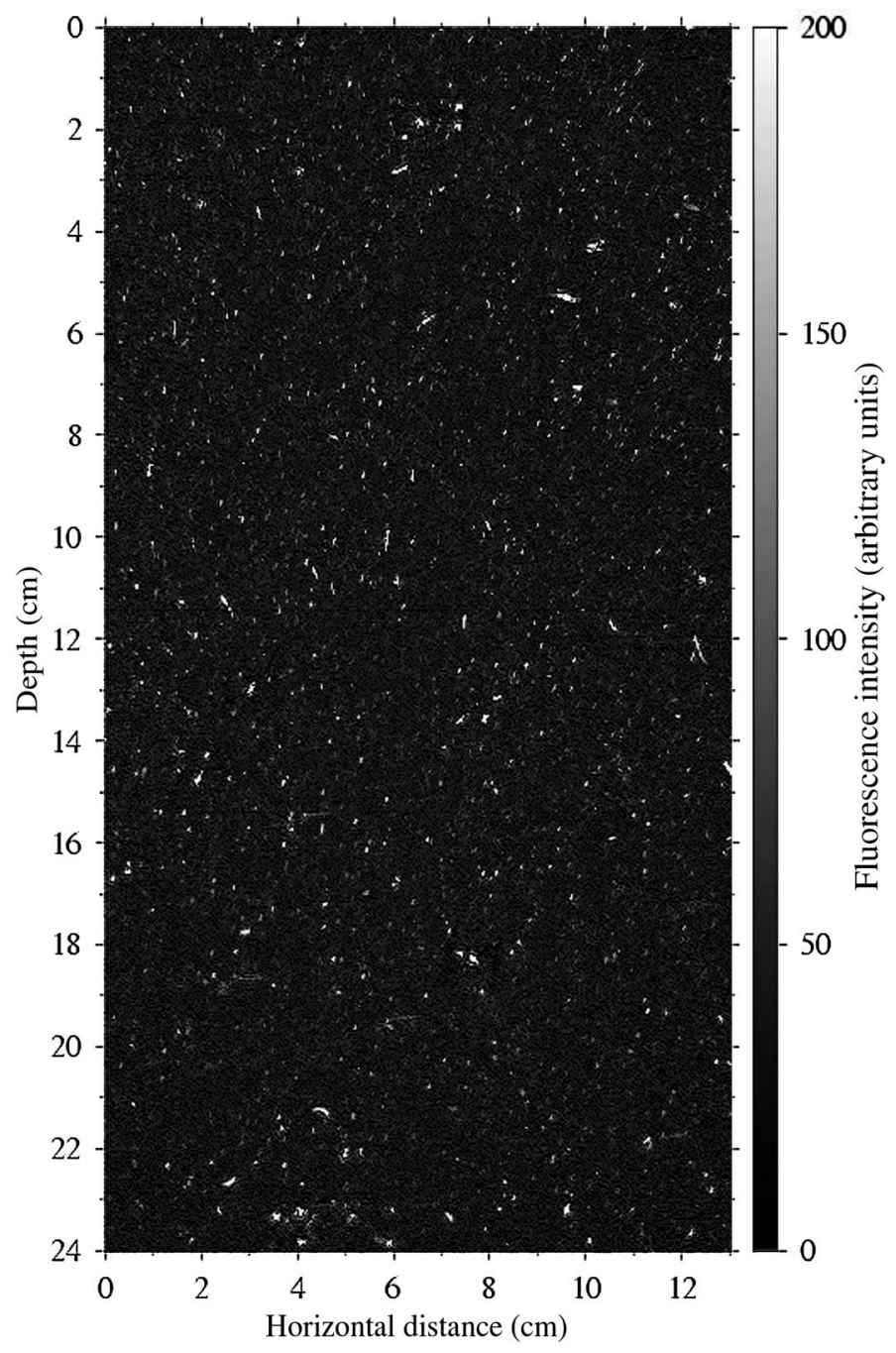

Fig. 4. A $24 \times 13 \mathrm{~cm}$ section from a $32 \times 32 \mathrm{~cm}$ image of phytoplankton fluorescence taken with an imaging fluorometer at about $35 \mathrm{~m}$ depth, $10 \mathrm{~km}$ off the coast of San Diego, CA, USA. The local chlorophyll a concentration was about $0.6 \mu \mathrm{g} \mathrm{l}^{-1}$. The bar at the right indicates the relative fluorescence of the objects. The image has been corrected for spreading of the incident laser beam. The resolution of the image is $300 \times$ $300 \mu \mathrm{m}$; the large fluorescent objects are large diatom chains, other phytoplankton, and aggregates 


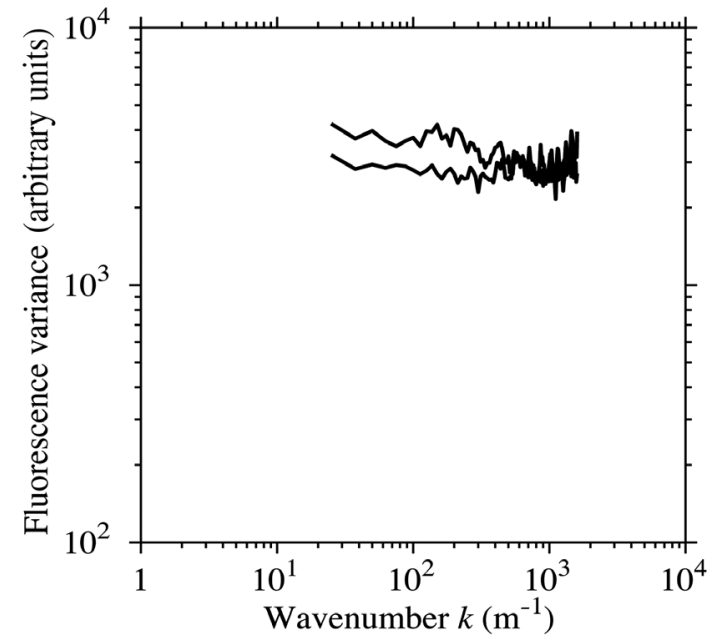

Fig. 5. Horizontal and vertical spatial spectra calculated from the image of Fig. 4. 256 pixel spectra were calculated for 400 rows or columns of the image, and then averaged to obtain 1 vertical and 1 horizontal spectrum. Even though the highwavenumber end of the inertial subrange would probably have been resolved in such an image, the spectra are white (flat) because of the spikes of fluorescence caused by large phytoplankton cells and aggregates (see Fig. 6)

Diego, CA, USA. The image shown in Fig. 4 was captured at the chlorophyll maximum layer $\left(\sim 0.6 \mu \mathrm{g} \mathrm{l}^{-1} \mathrm{chl}\right.$ a) at a depth of about $35 \mathrm{~m}$. The image has been corrected for the spreading of the incident laser beam, and a $24 \times 13 \mathrm{~cm}$ portion of the total image was extracted for analysis. The resolution of the image is $300 \times 300 \mu \mathrm{m}$, and individual phytoplankton cells can be clearly seen in the image (see also Zawada 2002).

The image of Fig. 4 is large enough $(24 \times 13 \mathrm{~cm})$ to resolve the high-wavenumber end of the inertial subrange, should it exist, suggesting that we might expect to see a $k^{-5 / 3}$ spectral slope of the phytoplankton fluorescence. To test this hypothesis, I calculated spectra from 400 rows and columns of the image, each row or column being 256 pixels $(7.7 \mathrm{~cm})$ long. I then averaged all the rows and all the columns (Fig. 5) to reduce the confidence limits of the spectrum. It is clear from the horizontal and vertical average spectra of Fig. 5 that there is no $k^{-5 / 3}$ region of the spectrum; in fact, the spectra have no slope at all. The spectrum is 'white'. Why is this?

A column of 800 pixels (fluorescence intensity versus distance) arbitrarily chosen from Fig. 4 is plotted in Fig. 6. It can be seen that the fluorescence is dominated by a few very intense spikes at the locations of large cells. These spikes behave as delta functions in the Fourier transform that generates the spectrum. The Fourier transform of a delta function is a constant in wavenumber space; that is, equal energy (variance, spectral density) at all wavenumbers. Thus, the few,

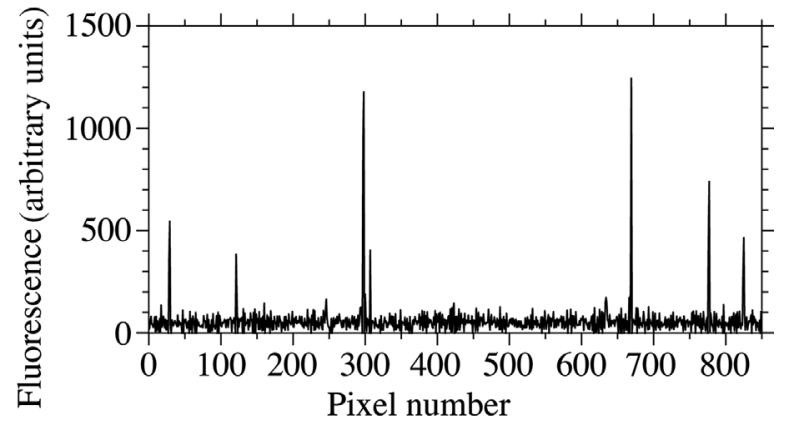

Fig. 6. An example of the fluorescence versus distance along a column of pixels taken from the image in Fig. 4. Note the large isolated spikes of fluorescence caused by individual cells

large, intensely fluorescent cells cause the spectrum to be flat, or white. As an example of this, I created 256 point data series with 20 randomly placed delta functions. I then calculated the spectrum of the series, and averaged the spectra of 400 such series (Fig. 7). The spectrum, as that of Fig. 5, is white.
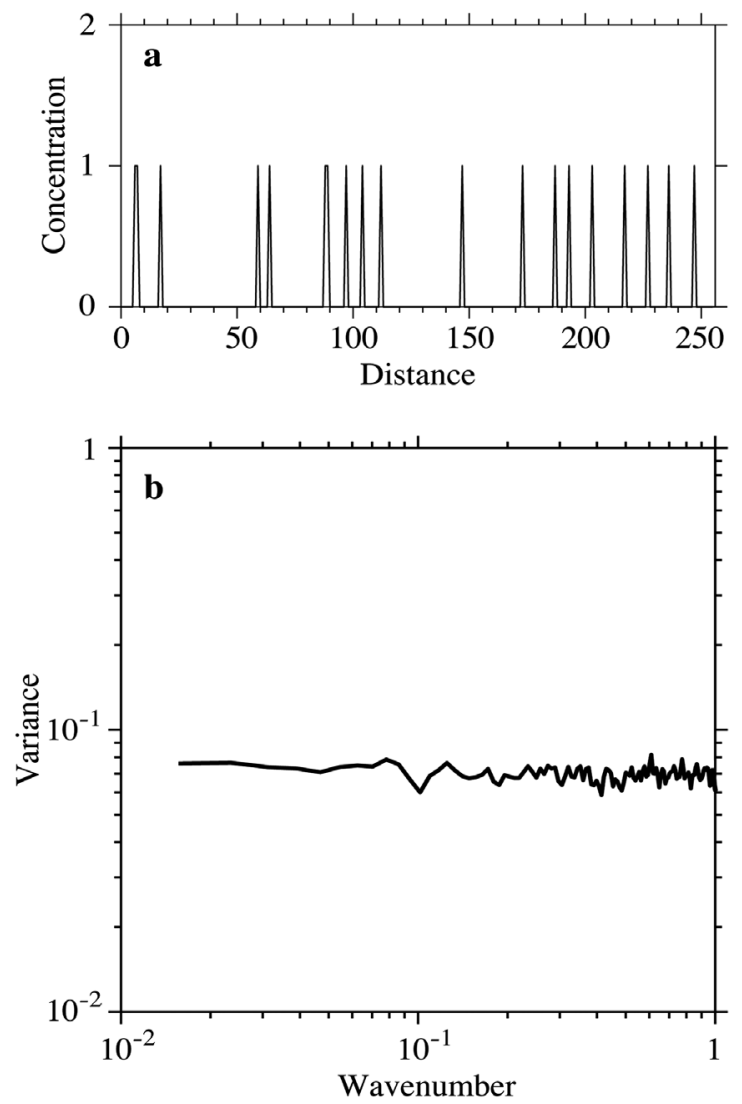

Fig. 7. (a) A 256 point data series composed of 20 delta functions. (b) The average of 400 spectra made up of data series such as that shown in (a). Compare the shape of this spectrum to those of Fig. 5. Units for all axes are arbitrary 
As plankton are quanta, the only way to obtain an underlying $k^{-5 / 3}$ spectrum from a data series of plankton is to average over such a large volume that individual plankters no longer contribute to the variance. The plankton can then be viewed as a continuum. How large a volume must we average over? As given in Siegel (1998), we will have about a $1 \%$ counting error when 400 cells are counted. Thus, to minimize the contribution of individuals, we must average over a volume of water that contains about 400 of the types of organisms whose individual fluorescence is intense enough to cause large quantum changes in the local fluorescence. Large, intensely fluorescent phytoplankton cells might have a concentration of 1 to 10 cells ml $^{-1}$, giving an averaging volume of 40 to $400 \mathrm{ml}$. The length scale that is associated with this volume depends on the instrument, but would usually be $>10 \mathrm{~cm}$, and probably $>50 \mathrm{~cm}$ in most cases. The length scales are much longer when counting zooplankton, as they are much rarer. These length scales suggest that we cannot resolve the high-wavenumber end of the inertial subrange using plankton, and that we can only resolve the low-wavenumber end when it occurs at relatively large scales $(>1 \mathrm{~m})$. This implies that we may only be able to find a turbulence-induced $k^{-5 / 3}$ spectral slope of plankton in extremely turbulent environments; for example, in the tidally mixed waters over Georges Bank. In more quiescent environments, we may be able to resolve a $k^{-5 / 3}$ spectrum of plankton if the plankton are dominated by abundant small cells; i.e., no spikes of fluorescence due to large individuals. One conclusion is that you need to know what you are sampling. Even a few spikes will cause a spectrum to become whiter (as discussed in more detail below).

\section{ALTERNATE MODELS OF PLANKTON PATCHINESS}

As shown above, most studies of plankton patchiness resolve scales that are larger than the inertial subrange of turbulence. It is, therefore, useful to explore the physical dynamics that might dominate at these scales, and to investigate the expected spectral slopes of a tracer embedded in these flows. The 2 types of physical dynamics I will consider are internal waves and 2D geostrophic turbulence.

\section{Internal waves}

Internal waves dominate the motions of stratified waters at frequencies lower than the buoyancy frequency $N$, and higher than the Coriolis frequency $f$. Motions at higher frequencies than $N$ are turbulent, and motions at lower frequencies than $f$ tend to be in a geostrophic balance. The spatial scales of internal waves range from $\sim 1 \mathrm{~m}$ to $\sim 5 \mathrm{~km}$. Internal waves can propagate in any direction, with lower-frequency waves propagating vertically, and high-frequency waves propagating horizontally (as a surface gravity wave).

The spectral slope of temperature variance in a welldeveloped internal wave field depends on the way in which it is measured (Phillips 1980). In horizontal transects, the wavenumber spectrum of temperature variance should fall as $k^{-5 / 2}$. In vertical profiling, the frequency spectrum should fall as $\omega^{-3}$, where $\omega$ is the frequency. Hence, the sampling scheme is an important determinant of the spectral slope, and transformations from frequency to wavenumber (time to space) based on, for example, ambient horizontal currents may give incorrect results.

For horizontal transects, a $k^{-5 / 2}$ slope is significantly steeper than a $k^{-5 / 3}$ slope, and should be distinguishable with sufficiently well-resolved data series. The steeper slope implies that there is relatively less variability at small scales in an internal wave field compared to 3D turbulence.

Piontkovski et al. (1997) calculated spectral slopes for temperature, phytoplankton (fluorescence), and zooplankton (pump samples), sampled with $5 \mathrm{~km}$ resolution along transects in the Indian and Atlantic Oceans, and the Mediterranean Sea. From the spectral slopes of $k^{-2}$ to $k^{-3}$, they concluded that the variability was driven by the low-frequency internal wave field, though patchiness created by geostrophic turbulence would also be consistent with these results (see below).

\section{Geostrophic turbulence}

At scales larger than the baroclinic Rossby radius of deformation ( $10 \mathrm{~km}$ in most of the ocean), and time scales longer than $f^{-1}$, motions of the water are largely horizontal and approximately 2D. The ambient fronts and eddies create a large-scale turbulence known as 2D 'geostrophic' turbulence (e.g. Kraichnan 1967, Charney 1971). This turbulence is fundamentally different than 3D turbulence, since tilting and stretching of vertical vortices are not possible in 2 dimensions.

In geostrophic turbulence, the velocity spectrum is predicted to fall as $k^{-3}$, while a passive tracer is predicted to have a $k^{-1}$ spectral slope (Kraichnan 1967, Charney 1971, Bennett \& Denman 1985). Over the long-time scales $\left(>f^{-1}\right)$ of geostrophic turbulence, plankton can no longer be considered passive tracers, as their growth and mortality create structures in the environment. As shown by Abraham (1998), the growth rates of the plankton are a strong determinant of the spectral slope; fast-growing organisms (e.g. 
phytoplankton) tend to have spectral slopes near $k^{-3}$, while slower-growing organisms (e.g. crustacean zooplankton) have spectral slopes near $k^{-1}$. The ratio of the biological timescale to the timescale of the flow determines the spectral slope (e.g. Abraham \& Bowen 2002), with slower-growing organisms behaving more like a passive tracer; higher variance at high wavenumbers (more structure at small scales).

Powell \& Okubo (1994) modeled both passive and reactive constituents of $2 \mathrm{D}$ and $3 \mathrm{D}$ turbulence. They found that non-reactive tracers behaved as predicted $\left(k^{-1}\right.$ spectral slope in 2D geostrophic turbulence). However, when the organisms were allowed to interact, the spectrum tended to flatten, becoming whiter, with relatively more variance at small scales. Washburn et al. (1998) calculated spectral slopes for temperature, salinity, chlorophyll fluorescence, and beam attenuation coefficient from data collected along transects in the subarctic North Atlantic. They found fluorescence and salinity to be coherent at scales $>7 \mathrm{~km}$, with a spectral slope of $k^{-2}$. At higher wavenumbers, the fluorescence spectrum fell off as $k^{-3}$, while the salinity spectrum continued as $k^{-2}$. They suggested
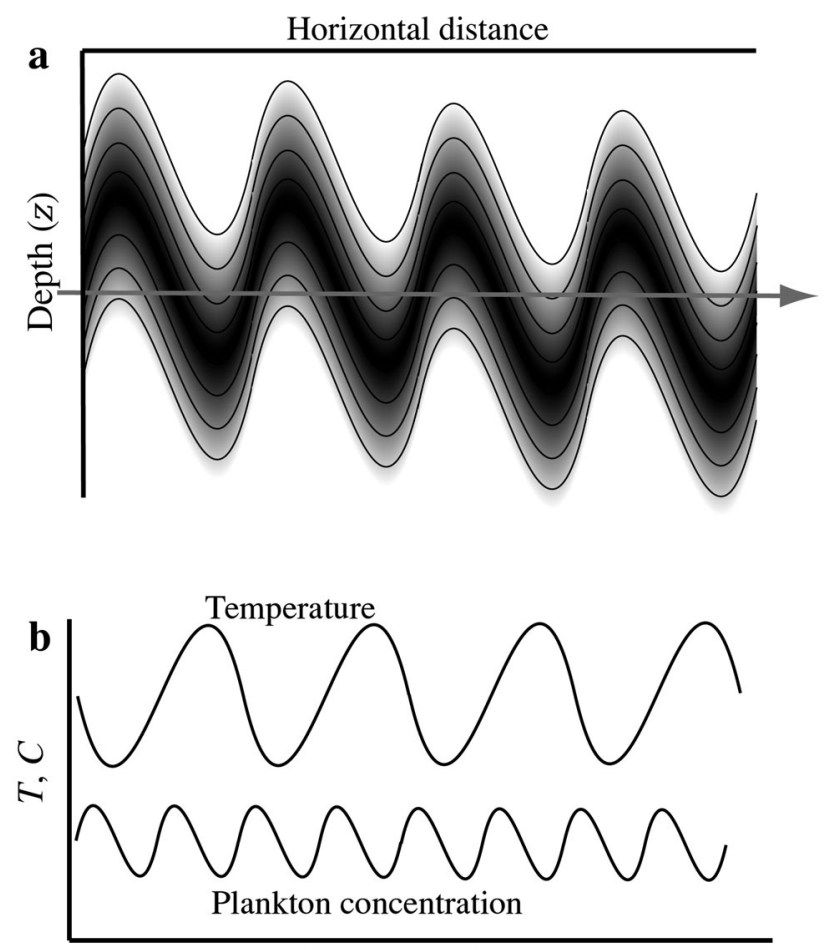

Horizontal distance

Fig. 8. (a) A depth-horizontal section of temperature $T$ (black lines) and a layer of plankton $C$ (gray scale). Temperature decreases monotonically from top to bottom, while the plankton does not. (b) Data series of $T$ and $C$ from a transect taken along the straight gray arrow in (a). As the plankton concentration is not monotonic, there is a higher wavenumber variability in plankton series $C$ than in the $T$ series that at low wavenumbers the variability was a result of horizontal stirring (geostrophic turbulence), while at higher wavenumbers, non-conservative biological processes dominated. Smith et al. (1988) analyzed satellite images of phytoplankton pigment and found spectral slopes of about $k^{-3}$ offshore, $k^{-2.2}$ inshore, and $k^{-1}$ slopes at length scales $<10 \mathrm{~km}$. These results are broadly consistent with stirring of reactive tracers by geostrophic turbulence, though distinguishing the underlying dynamics (internal waves versus geostrophic turbulence) based solely on the spectral slope would be impossible.

\section{WHY ARE THE PHYSICAL AND BIOLOGICAL SPECTRAL SLOPES DIFFERENT?}

Even though they have not sampled the inertial subrange, many studies have found spectral slopes of plankton to be close to $k^{-5 / 3}$. Furthermore, it is a common observation that the spectral slopes of the plankton are different (usually less steep) than the slopes of hydrographic properties such as temperature. These differences are generally interpreted in terms of the relative dominance of physical and biological dynamics. However, there are properties of the plankton that may predispose them to having different spectral slopes than temperature, and to have spectral slopes near $k^{-5 / 3}$ even though the underlying physical dynamics have nothing to do with 3D isotropic turbulence. Here I consider 4 potential mechanisms that will give a planktonic spectral slope that is different from the velocity or temperature spectra: (1) layers, (2) spikes, (3) steps, and (4) trends.

\section{Layers}

In the ocean, temperature tends to decrease monotonically from the surface to depth due to its effect on density. Phytoplankton, on the other hand, are not thus constrained, and can often be found in isolated layers, including the deep chlorophyll maximum. Horizontal transects through a temperature/phytoplankton field that is deformed by internal waves can thus give quite different results for the variability of the 2 fields (Fig. 8). A thin layer of phytoplankton that is deformed by an internal wave will create maxima and minima of fluorescence twice as often as maxima and minima of temperature. This will create high-wavenumber variability in the phytoplankton that is not present in the temperature, causing the fluorescence spectrum to be flatter at high wavenumbers than the temperature spectrum. Thus, instead of the $k^{-5 / 2}$ spectrum we would expect for temperature in a well-developed 
internal wave field, we might find the fluorescence spectrum to be flatter, perhaps closer to $k^{-5 / 3}$ than $k^{-5 / 2}$.

A further problem with using fluorescence as a proxy for patchiness of phytoplankton biomass is that fluorescence is a complicated, non-linear and time-dependent function of the cell's light history. At time scales shorter than about $15 \mathrm{~min}$, the fluorescence yield (fluorescence as a function of irradiance) decreases with increasing irradiance above about $100 \mu$ Ein $\mathrm{m}^{-2} \mathrm{~s}^{-1}$ due to non-photochemical quenching (e.g. Morrison 2003 and references therein). Cells that are moved upward, due to mixing or high-frequency internal waves, and then are moved down again will have a lower intensity of fluorescence than cells that were stationary in the light field. Thus, high-frequency vertical motions of

the water can create horizontal variations in fluorescence that are uncoupled from variations in biomass, or even the present location of the isopycnals (e.g. Lennert-Cody \& Franks 2002). Over longer time scales, the fluorescence yield changes due to photoadaptation, a process that may also introduce spatial variability of fluorescence to an otherwise homogeneous biomass distribution.

\section{Spikes}

As discussed above, plankton is made up of individuals, some of which can be quite large or intensely fluorescent. A few large individuals in a time series of fluorescence, for example, will create a time series

b
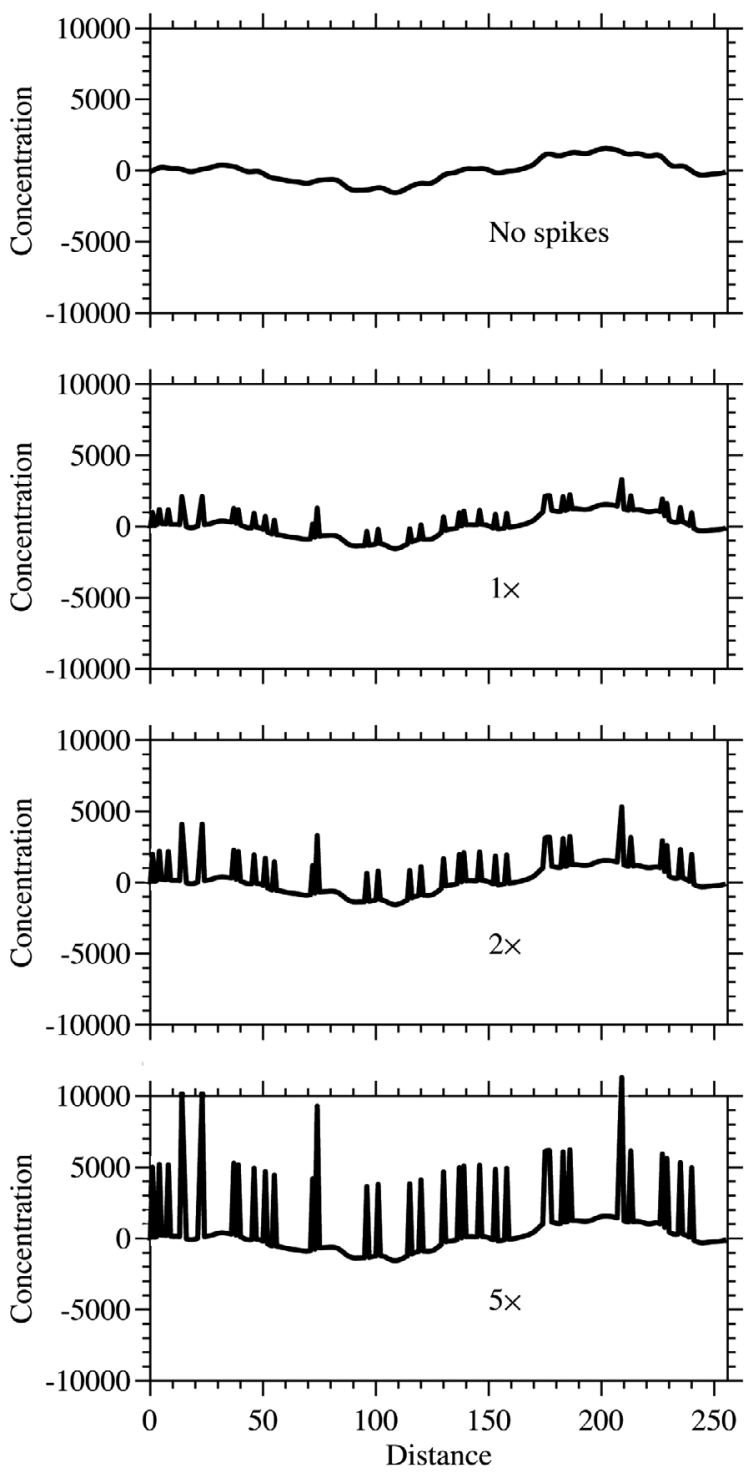
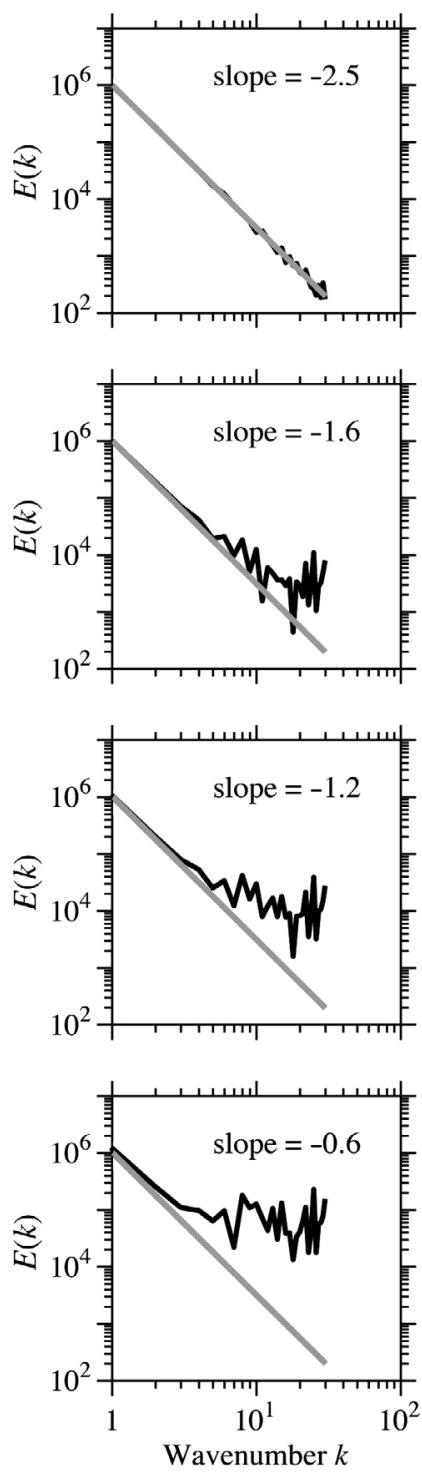

dominated by isolated spikes. These isolated spikes behave as delta functions, and the Fourier transform of a delta function is a constant; that is, a white spectrum. Thus, the inclusion of a few spikes in the data will tend to shift any underlying spectral distribution towards a flat (white) spectrum.

To illustrate this effect, I created a synthetic data set from 30 sine waves whose amplitude was determined by a $k^{-5 / 2}$ spectral slope, characteristic of internal waves. The sine waves were assigned a randomly chosen phase (the relative positions of the crests of the waves), and a data set 256 points long was created. To this data set, I added 40 randomlyplaced spikes that had an amplitude $0,1,2$, or $5 \times$ the amplitude of the largest (lowest wavenumber)

Fig. 9. (a) Synthetic data sets made of 30 sine waves with random phase and an underlying $k^{-5 / 2}$ spectrum. Added to these data are delta functions of size (top-to-bottom) $0,1,2$ and $5 \times$ the amplitude of the lowest wavenumber sine wave. (b) $k^{-5 / 2}$ spectral slope (gray line) and spectrum calculated from data series in (a) (black line). The spectral slope of the data is calculated by linear regression of the log of the spectrum and the log of the wavenumber. Note that the high-wavenumber component of the spectrum is most strongly affected by the inclusion of spikes in the data. Units for all axes are arbitrary 
sine wave (Fig. 9). I then calculated the spectral slope of the data sets after removing any linear trend. There is a clear tendency for the spikes to lower the slope of the spectrum, from $k^{-5 / 2}$ with no spikes, to $\sim k^{-2 / 3}$ when the spikes were $5 \times$ the amplitude of the largest sine wave.

Spikes in plankton could arise from any number of causes that have nothing to do with mixing of a dissolved tracer in 3D isotropic turbulence. Fluorescence spikes could be created by large cells, aggregates, pieces of seaweed, zooplankton guts, or even something getting temporarily jammed in the instrument. Careful examination of the raw data, and in particular visual examination of water samples, is essential for assessing whether the spikes should be interpreted or not. The occurrence of spikes and the consequent differences between the temperature and planktonic spectra may have little to do with the relative strength of the underlying physical and biological dynamics, the usual explanation for such differences.

\section{Steps}

A step or front is a sudden change in the local average value of a property. Steps and fronts are common features of the ocean, and occur in both physical and biological variables, though not always coincidently. The spectrum of a step gives a $k^{-2}$ slope. Any data set containing steps (sharp fronts), regardless of its underlying physically driven spectral slope, will tend toward a spectral slope of $k^{-2}$.

An example of the influence of steps on the spectral slope can be easily formulated. I took 30 sine waves with random phase, all with equal amplitudes (an underlying flat, white spectrum). I then added 4 randomly placed steps of random sign (positive or negative steps), with amplitudes of $0,1,10$ or $20 \times$ the amplitude of the sine waves (Fig. 10). The spectra calculated from these data series clearly show the tendency of the steps to force the spectral slope toward $k^{-2}$. With steps $10 \times$ the amplitude of the underlying sine waves, the spectral slope is already $k^{-1}$, though the steps are not easy to pick out in the data.

Steps or fronts in the data could thus make an underlying white spectrum tend toward $k^{-5 / 3}$, or a spectrum of a tracer in an internal wave field $\left(k^{-5 / 2}\right)$ tend toward $k^{-2}$ or $k^{-5 / 3}$. Washburn et al. (1998) rec-

Fig. 10. (a) Synthetic data sets made of 30 sine waves with random phase and an underlying $k^{0}$ (white) spectrum. Added to these data are steps of size (top-to-bottom) 0, 1, 10 and $20 \times$ the amplitude of the sine waves. (b) $k^{0}$ spectral slope (gray line) and spectrum calculated from data series in (a) (black line). Spectral slope of data is calculated by linear regression of the log of the spectrum and the log of the wavenumber. Units for all axes are arbitrary 


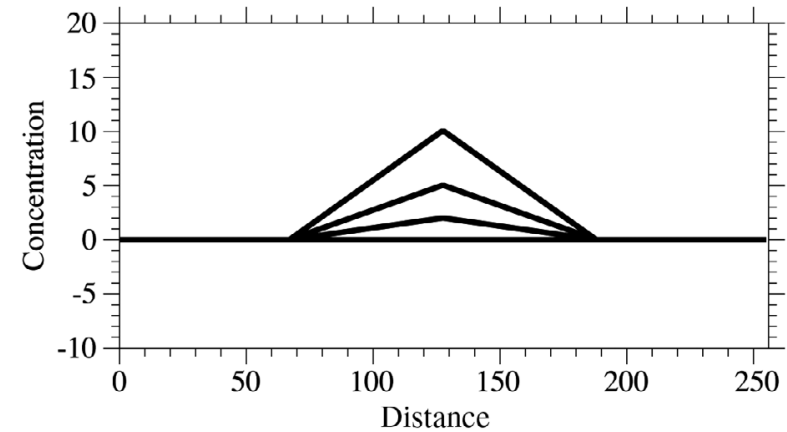

Fig. 11. Linear ramps used to explore the effects of this feature on the spectral slope of the data. Four ramps were used (bottom-to-top): $0,2,5$, and $10 \times$ the amplitude of the sine waves (see Fig. 12). Units for both axes are arbitrary ognized this effect of steps, and removed a large step from their data prior to calculating the spectral slopes (which ended up being about $k^{-2}$ in any case).

\section{Trends}

In a fine paper that presaged this one by almost 2 decades, Armi \& Flament (1985) discuss the effect of linear trends in a data series. They showed that a peak made up of 2 linear ramps (increasing and decreasing) has a Fourier transform that gives a spectrum that falls off as $k^{-4}$. A data series with such a linear trend would have a slope tending towards $k^{-4}$.

Following the approach of the previous sections, I made a synthetic data series of 30 sine waves with random phase, all with the same amplitude (an underlying white spectrum). I then added a linear ramp (Fig. 11) to the data series, the peak of the ramp being $0,2,5$, or $10 \times$ the amplitude of the sine waves. The resulting spectra clearly show the effects of the ramp (Fig. 12), even though the ramp is difficult to see in the data. As the ramp gets taller, the spectrum falls off more steeply, reaching $k^{-2}$ (for the low-wavenumber part of the spectrum) when the ramp is $10 \times$ the sine wave amplitude. There is also a marked change in the slope of each spectrum from negative slopes at low wavenumbers to zero slope at higher wave numbers. The break occurs at the same scale as the ramp width (120 points).

The inclusion of a ramp-like trend in the data could easily drive an

Fig. 12. (a) Synthetic data sets made of 30 sine waves with random phase and an underlying $k^{0}$ (white) spectrum. Added to these data are ramps of amplitude (topto-bottom) $0,2,5$ and $10 \times$ the amplitude of the sine waves (see Fig. 11). (b) $k^{0}$ spectral slope (gray line) and spectrum calculated from data series in (a) (black line). The spectral slope of the data is calculated by linear regression of the log of the spectrum and the log of the wavenumber for only the lowest 5 wavenumbers. Note that it is the lowest wavenumbers that are most strongly affected by the presence of a ramp. Units for all axes are arbitrary 
underlying white spectrum to have a $k^{-5 / 3}$ slope, particularly at the lower wavenumbers. The important question to ask is how the ramp in the data got there. Is it a result of the instrument, the sampling, or the underlying physical and biological dynamics?

\section{What does $k^{-5 / 3}$ look like?}

I have given 4 distinct ways of generating a $k^{-5 / 3}$ spectrum: (1) a combination of sine waves of the appropriate amplitudes, (2) a combination of sine waves and spikes in the data, (3) a combination of sines and steps, and (4) a combination of sines and linear ramps. To show how different these data series look, I created synthetic data sets, each of which has a $k^{-5 / 3}$ spectral slope (Fig. 13). These data sets were made up of (1) pure sine waves with amplitudes giving a $k^{-5 / 3}$ slope, (2) a set of sine waves with a white spectrum plus 7 steps of random sign and magnitude, (3) a set of sine waves with a $k^{-5 / 2}$ spectrum (characteristic of internal waves) with randomly placed spikes of random magnitude, and (4) a random combination of steps $\left(k^{-2}\right)$ and spikes $\left(k^{0}\right)$. All these data sets have a $k^{-5 / 3}$ spectrum, yet they look very different when plotted as the raw data series.

When we calculate a spectrum of a data series, we throw away some essential information; the phase relationships of the various Fourier components making up the spectrum (see also Armi \& Flament 1985, for further analysis of this issue). The data sets of Fig. 13 all have the same spectrum, but very different phase relationships of the various sines and cosines making up the Fourier decomposition of the data. It is this relative phase that gives the data set its visual character. It is vitally important, then, to spend some time looking at the raw data to try to identify the features of the data that might be driving the spectral slope, and ask whether these features are included in the dynamics of the model that the data are being compared to. Large spikes in the fluorescence data may indicate rare and random pieces of detritus that cannot be accommodated in a continuum model of a reactive tracer in a turbulent flow. Large steps in the fluorescence may indicate changes in species composition with consequent changes in the fluorescence yield, but may have little to do with the underlying physical flows. In general, there is not enough information in the spectral slope to unambiguously identify the dynam-

Fig. 13. Synthetic data series, all with a $k^{-5 / 3}$ spectral slope. (a) 30 sine waves of random phase, (b) 30 sine waves with a $k^{0}$ (white) spectrum with 7 randomly placed steps of random sign and magnitude, (c) 30 sine waves with a $k^{-5 / 2}$ (internal wave) spectrum with 40 randomly placed spikes, and (d) a combination of steps $\left(k^{-2}\right)$ and spikes $\left(k^{0}\right)$. Units for both axes are arbitrary 
ics that created the patterns. The spectral slope is not a strong test of any model of physical-biological interactions, and should only be used as a supplement to other careful analyses.

\section{CONCLUSIONS}

Theoretical models predicting the spectrum of plankton patchiness offer the potential of a mechanistic understanding of the physical and biological dynamics structuring the distributions. The $k^{-5 / 3}$ model of plankton advected in a 3D isotropic turbulent flow is often the basis for comparison to field data. However, an analysis of recent publications suggests that most field data do not resolve the appropriate spatial scales to allow comparison of the field data to the $k^{-5 / 3}$ model. Furthermore, it may be impossible in most cases to gather planktonic data for testing the spectral slope in the inertial subrange of turbulence because of the quantum character of the plankton. Plankton are not a continuum as modeled, but are individuals. To remove the effect of individuals from the data (since individuals would tend to make the spectrum flatter and more white), some averaging must be performed. The scales for averaging will depend on the types of organisms present, so some knowledge of the composition of the plankton is necessary. In many cases, the averaging scales will be so large that the inertial subrange cannot be resolved with planktonic data. It is only when the effects of individual plankton are undetectable, and the inertial subrange is sufficiently large (e.g. intense turbulence and low stratification) that planktonic data can be robustly compared to the $k^{-5 / 3}$ spectral model.

Most planktonic data are gathered at spatial scales that are more appropriate for comparison to the internal wave $\left(k^{-5 / 2}\right.$ for transects) or geostrophic turbulence ( $k^{-3}$ to $k^{-1}$ for reactive tracers) spectral models. Still, the biological spectra often differ from these models. These differences could arise through the biological dynamics that are usually invoked. However, other factors such as spikes in the data, sharp steps in the data, layers of plankton, or linear ramps in the data can confound the interpretation of the underlying spectrum. Calculation of the spectrum necessitates throwing away important information concerning the phase relationships of the Fourier components of the data. It is the phase, not the amplitude, which determines much of the visual character of the data. The data should be carefully examined for such features, and decisions made about whether their effects on the spectral slope are consistent with the model that the data are being compared to. The spectral slope, by itself, does not contain enough information to unambiguously identify the dynamics underlying the observations, or even to distinguish among different spectral models. It is important to be critical when comparing models and data: (1) Does the model contain the appropriate dynamics? (2) Do the data test the model? (3) Are the data of the correct dimensionality for comparison to the model? Careful consideration of these factors may help us develop new and powerful insights into the dynamics coupling physics and biology in the ocean.

Acknowledgements. This paper has been a long time brewing, and has benefited from discussions with many people. While I acknowledge their considerable help in synthesizing these ideas, I must emphasize that I am solely culpable for any mistakes, distortions, misstatements etc., that may appear in this manuscript. In particular, I wish to thank K. Fisher, J. Jaffe, D. Rudnick, J. Pringle, W. Munk, C. Garrett, A. Gargett, E. Boss, and H. Yamazaki for fruitful discussions and occasional flashes of insight. This work was supported by NSF grants DBI-9871359 and OCE02-20379, and ONR grant N00014-03-1-0391.

\section{LITERATURE CITED}

Abraham ER (1998) The generation of plankton patchiness by turbulent stirring. Nature 391:577-580

Abraham ER, Bowen MM (2002) Chaotic stirring by a mesoscale surface-ocean flow. Chaos 12:373-381

Armi L, Flament P (1985) Cautionary remarks on the spectral intepretation of turbulent flows. J Geophys Res 90: 11779-11782

Batchelor GK (1959) Small-scale variation of convected quantities like temperature in turbulent fluid. J Fluid Mech 5: 113-133

Bennett AF, Denman KL (1985) Phytoplankton patchiness: inferences from particle statistics. J Mar Res 43:307-335

Charney JG (1971) Geostrophic turbulence. J Atmos Sci 28: $1087-1095$

Corrsin S (1961) The reactant concentration spectrum in turbulent mixing with a first-order reaction. J Fluid Mech 11: 407-416

Denman KL, Platt T (1976) The variance spectrum of phytoplankton in a turbulent ocean. J Mar Res 34:593-601

Denman KL, Okubo A, Platt T (1977) The chorophyll fluctuation spectrum in the sea. Limnol Oceanogr 22:1033-1038

Franks PJS, Jaffe JS (2001) Microscale distributions of phytoplankton: initial results from a two-dimensional imaging fluorometer, OSST. Mar Ecol Prog Ser 220:59-72

Gargett AE (1985) Evolution of scalar spectra with the decay of turbulence in a stratified flow. J Fluid Mech 159: $379-407$

Gargett AE, Osborn TR, Nasmyth PW (1984) Local isotropy and the decay of turbulence in a stratified fluid. J Fluid Mech 144:231-280

Hinze J (1959) Turbulence. McGraw-Hill, New York, NY

Kolmogorov AN (1941) The local structure of turbulence in an incompressible viscous fluid for very large Reynolds number. CR Acad Sci USSR 30:301-305

Kraichnan R (1967) Inertial ranges in two-dimensional turbulence. Phys Fluids 10:1417-1423 
Lazier JRN, Mann KH (1989) Turbulence and the diffusive layers around small organisms. Deep-Sea Res 36: 1721-1733

Lennert-Cody CE, Franks PJS (2002) Fluorescence patches in high-frequency internal waves. Mar Ecol Prog Ser 235: $29-42$

Lovejoy S, Currie WJS, Tessier Y, Claereboudt MR, Bourget E, Roff JC, Schertzer D (2001a). Universal multifractals and ocean patchiness: phytoplankton, physical fields and coastal heterogeneity. J Plankton Res 23:117-141

Lovejoy S, Schertzer D, Tessier Y, Gaonac'h H (2001b). Multifractal and resolution-independent remote sensing algorithms: the example of ocean colour. Int J Remote Sens 22: 1191-1234

Martin AP, Srokosz MA (2002) Plankton distribution spectra: inter-size class variability and the relative slopes for phytoplankton and zooplankton. Geophys Res Lett 29, paper 2213

Morrison JR (2003) In situ determination of the quantum yield of phytoplankton chlorophyll a fluorescence: a simple algorithm, observations, and a model. Limnol Oceanogr 48:618-631

Mountain DG, Taylor MH (1996) Fluorescence structure in the region of the tidal mixing front on the southern flank of Georges Bank. Deep-Sea Res II 43:1831-1853

Pershing AJ, Wiebe PH, Manning JP, Copley NJ (2001) Evidence for vertical circulation cells in the well-mixed area of Georges Bank and their biological implications. DeepSea Res II 48:283-310

Phillips OM (1980) The dynamics of the upper ocean. Cambridge University Press, Cambridge

Piontkovski SA, Williams R, Peterson WT, Yunev OA, Minkina NI, Vladimirov VL, Blinkov A (1997) Spatial heterogeneity of the planktonic fields in the upper mixed layer of the open ocean. Mar Ecol Prog Ser 148:145-154

Powell TM, Okubo A (1994) Turbulence, diffusion and patchiness in the sea. Phil Trans R Soc Lond B 343:11-18

Seuront L, Lagadeuc Y (1997) Characterisation of space-time variability in stratified and mixed coastal waters (Baie des Chaleurs, Québec, Canada): application of fractal theory. Mar Ecol Prog Ser 159:81-95

Seuront L, Lagadeuc Y (2001) Multiscale patchiness of the calanoid copepod Temora longicornis in a turbulent

Editorial responsibility: Otto Kinne (Editor-in-Chief), Oldendorf/Luhe, Germany coastal sea. J Plankton Res 23:1137-1145

Seuront L, Schmitt F, Lagadeuc Y, Schertzer D, Lovejoy S (1996a) Multifractal analysis of phytoplankton biomass and temperature in the ocean. Geophys Res Lett 23:3591-3594

Seuront L, Schmitt F, Schertzer D, Lagadeuc Y, Lovejoy S (1996b) Multifractal intermittency of Eulerian and Lagrangian turbulence of ocean temperature and plankton fields. Nonlinear Proc Geophys 3:236-246

Seuront L, Schmitt F, Lagadeuc Y, Schertzer D, Lovejoy S (1999) Universal multifractal analysis as a tool to characterize multiscale intermittent patterns: example of phytoplankton distribution in turbulent coastal waters. J Plankton Res 21:877-922

Seuront L, Gentilhomme V, Lagadeuc Y (2002) Small-scale nutrient patches in tidally mixed coastal waters. Mar Ecol Prog Ser 232:29-44

Siegel DA (1998) Resource competition in a discrete environment: Why are plankton distributions paradoxical? Limnol Oceanogr 43:1133-1146

Smith RC, Zhang X, Michaelsen J (1988) Variability of pigment biomass in the California Current System as determined by satellite imagery 1 . Spatial variability. J Geophys Res 93:10863-10882

Tsuda A, Sugisaki H, Ishimaru T, Saino T, Sato T (1993) White-noise-like distribution of the oceanic copepod Neocalanus cristatus in the subarctic North Pacific. Mar Ecol Prog Ser 97:39-46

Washburn L, Emery BM, Jones BH, Ondercin DG (1998) Eddy stirring and planktonic patchiness in the subarctic North Atlantic in late summer. Deep-Sea Res I 45:1411-1439

Wiebe PH, Mountain DG, Stanton TK, Greene CH, Lough G, Kaartvedt S, Dawson J, Copley N (1996) Acoustical study of the spatial distribution of plankton on Georges Bank and the relationship between volume backscattering strength and the taxonomic composition of the plankton. Deep-Sea Res II 43:1971-2001

Yoshida J, Oakey NS (1996) Characterization of vertical mixing at a tidal-front on Georges Bank. Deep-Sea Res II 43: 1713-1744

Zawada DG (2002) The application of a novel multispectral imaging system to the in vivo study of fluorescent compounds in selected marine organisms. PhD thesis, University of California, San Diego

Submitted: July 15, 2004; Accepted: January 3, 2005

Proofs received from author(s): May 20, 2005 